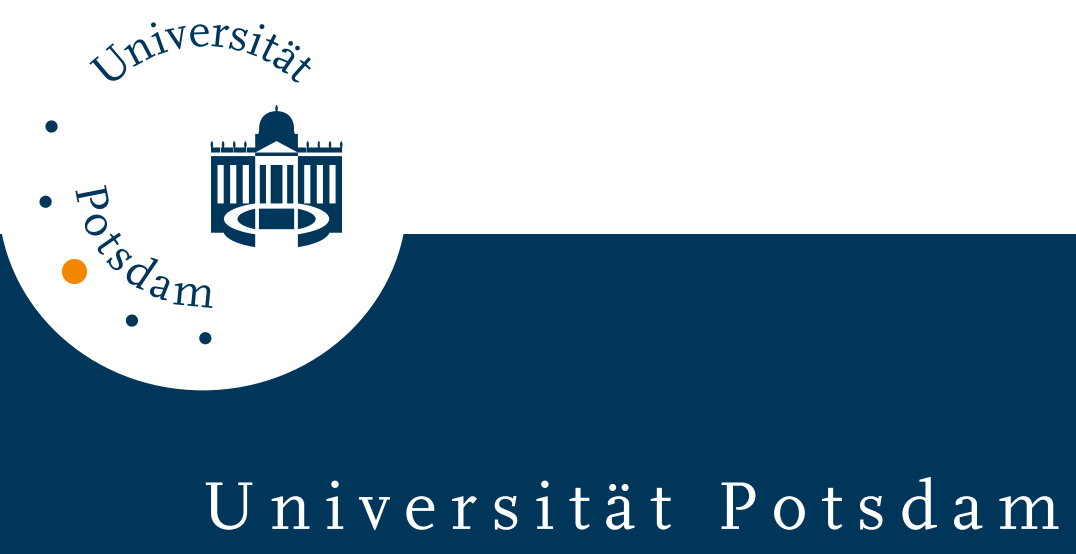

Martin Rolfs, Reinhold Kliegl, Ralf Engbert

\title{
Microsaccade orientation supports attentional enhancement opposite to a peripheral cue: Commentary on Tse, Sheinberg, and Logothetis
}

first published in:

Psychological Science. - ISSN 0956-7976. - 15 (2004), 10, S. 705 - 707

Postprint published at the Institutional Repository of the Potsdam University: In: Postprints der Universität Potsdam

Humanwissenschaftliche Reihe ; 248

http://opus.kobv.de/ubp/volltexte/2011/5708/

http://nbn-resolving.de/urn:nbn:de:kobv:517-opus-57081

Postprints der Universität Potsdam

Humanwissenschaftliche Reihe ; 248 



\title{
MICROSACCADE ORIENTATION SUPPORTS ATTENTIONAL ENHANCEMENT OPPOSITE TO A PERIPHERAL CUE: \\ Commentary on Tse, Sheinberg, and Logothetis
}

\author{
Martin Rolfs, Ralf Engbert, \& Reinhold Kliegl \\ University of Potsdam, Germany
}

Manuscript number: MS3-382

Word count: 996

Address correspondence to

Martin Rolfs

Department of Psychology

University of Potsdam

P.O. Box 601553

14415 Potsdam

Germany

email: rolfs@rz.uni-potsdam.de 
Tse, Sheinberg, and Logothetis (2003) exploited a 'change blindness' paradigm to map the redistribution of spatial attention in response to a peripherally flashed cue. The probability of change detection at a given location was used as a measure of attention allocation. Using this measure, a "hot spot" of attention (i.e., close to perfect change detection) was found along the cue-fixation axis. This hot spot extended in the hemifield opposite to the cued location. Here we show that an analysis of fixational eye movements in a spatial cuing paradigm supports this important finding.

During fixation of a stationary target, micromovements of the eyes occur involuntarily. These “fixational” eye movements are classified as drift, tremor, and microsaccades. Microsaccades are the fastest of these three components with amplitudes typically smaller than $1^{\circ}$ of visual angle. Recently, Engbert and Kliegl (2003b) demonstrated that microsaccades are modulated by visual attention using a classical spatial cuing paradigm (Posner, 1980) with central arrow cues indicating the most probable location of a later target. About 300 ms after cue presentation, the angular distribution of microsaccades was oriented towards the expected target location.

With pilot data from a larger experimental study, we examined the impact of peripheral cues on microsaccade rate and orientation. Using peripheral flashes - similar to the uninformative stimuli employed by Tse et al. (2003) - we found an orientation shift of microsaccades in the direction opposite to the cued location. Taken together with our results on the coupling of visuospatial attention and microsaccades (Engbert \& Kliegl, 2003b), these results are in agreement with attentional enhancement opposite to the cued location observed by Tse et al. (2003).

Method. Figure 1a illustrates the experimental procedure. Twenty-two participants performed 120 trials each. They fixated a central spot (size: $0.78^{\circ}$ ) during the entire trial and responded only with key presses. A cue (i.e., a 100-ms flash) was presented either to the left or to the right indicating the probable position (validity $=80 \%$ ) of a subsequent target stimulus. 
Manual response latencies to targets yielded benefits from valid cues, i.e. target at cue location, and costs from invalid cues, i.e. targets were presented opposite the cue locations. Fixation accuracy was checked with an EyeLink-II system (SR-Research) with a sampling rate of $500 \mathrm{~Hz}$ and an instrument spatial resolution better than $0.005^{\circ}$. Microsaccades were detected based on a transformation of eye movements to two-dimensional velocity space (Engbert and Kliegl, 2003b). During data preprocessing, trials with missing data (blinks) or saccades with amplitudes larger than $1^{\circ}$ were discarded. Final data analysis was based on 1678 trials from all participants (Fig. 1b).

Results. To detect changes in the angular distribution of microsaccades induced by the cues, we computed microsaccade rates (i.e., number of microsaccades per second) separately for orientations to the right and to the left. All microsaccades with a maximum angular deviation between $-45^{\circ}$ and $+45^{\circ}$ from the target direction were considered, since binocular microsaccades are predominantly horizontally oriented (Engbert \& Kliegl, 2003a). Figure 1b displays the change in microsaccade rates after left and right cues in the top and bottom panel, respectively. Rates were computed for each participant using a moving time window of 200 ms and then averaged over all participants; shaded areas indicate between-subjects standard errors. There was no significant difference between left-oriented and right-oriented microsaccade rates in the pre-cue intervals, but there was a significant excess of microsaccades with orientations opposite to cue direction in a time-window of about 250 to $400 \mathrm{~ms}$ after cue presentation, as reflected in a separation of rate curves by more than two standard errors. Given the coupling of visuospatial attention and microsaccade orientation (Engbert \& Kliegl, 2003b), we conclude that visual attention was enhanced in a direction opposite to cue location. Moreover, also considering the delay of saccadic eye movements following shifts of attention, the time window was in good agreement with the duration of the flash-target intervals of $176 \mathrm{~ms}$ and $247 \mathrm{~ms}$, where Tse et al. (2003) found the most pronounced effect of attentional enhancement opposite to the cued location. 
Discussion. These findings support Tse et al.'s (2003) results with a different experimental paradigm and dependent variable. Interestingly, Tse, Sheinberg, and Logothetis (2002) did not find differences in fixational eye-movements for changes induced by a peripheral flash in their paradigm between flash and non-flash conditions. Their paradigm included five display changes within a trial duration of less than 1.5 seconds, where stimulus onset asynchronies between cue and target ranged from 12 ms to 447 ms. High-acuity instructions (Bridgeman \& Palca, 1980; Winterson \& Collewijn, 1976) as well as display changes (Reingold \& Stampe, 2000; Engbert \& Kliegl, 2003b) decrease the rate of microsaccades. Consequently, a rapid sequence of five display changes will induce a strong inhibition of microsaccades. This suppression of microsaccades might preclude the observation of modulations in fixational eye movement statistics, as reported by Tse et al. (2002), albeit, modulations of neural activity related to oculomotor neurons might still be present.

How could microsaccadic oculomotor activity underlying covert shifts of visuospatial attention explain attentional enhancement opposite to a cued location? To maintain fixation during and after cue onset, participants must inhibit (automatic) saccadic reactions towards exogenous cues. The population coding of saccade orientations in the superior colliculus (Lee, Rohrer, \& Sparks, 1988) suggests that this inhibition results in a bias of activation of oculomotor neurons for movements to the opposite hemifield (see also Munoz \& Istvan, 1998). Moore and Fallah (2001) showed that microstimulation of oculomotor neurons summoned attention to the visual space represented by the cortical stimulation site even when the stimulation was too weak to elicit any large-scale oculomotor activity. Obviously, then, oculomotor activity (such as shown in our study) can result in a hot spot of attention opposite to a cued location as observed by Tse et al. (2003). 


\section{References}

Bridgeman, B., \& Palca, J. (1980). The role of microsaccades in high acuity observational tasks. Vision Research, 20, 813-817.

Engbert, R., \& Kliegl, R. (2003a). Binocular coordination in microsaccades. In J. Hyönä, R. Radach, \& H. Deubel (Eds.), The mind's eyes: Cognitive and applied aspects of eye movements. Oxford: Elsevier, pp. 103-117.

Engbert, R., \& Kliegl, R. (2003b) Microsaccades uncover the orientation of covert attention. Vision Research, 43, 1035-1045.

Lee, C.K., Rohrer, W.H., \& Sparks, D.L. (1988). Population coding of saccadic eyemovements by neurons in the superior colliculus. Nature, 332, 357-360.

Moore T., \& Fallah, M. (2001).Control of eye movements and spatial attention. Proceedings of the National Academy of Sciences, U.S.A., 98, 1273-1276.

Munoz, D.P., \& Istvan, P.J. (1998). Lateral inhibitory interactions in the intermediate layers of the monkey superior colliculus. Journal of Neurophysiology, 79, 1193-1209.

Posner, M.I. (1980). Orientation of attention. The VIIth Sir Frederic Bartlett lecture. Quarterly Journal of Experimental Psychology, 32A, 3-25.

Reingold, E.M., \& Stampe, D.M. (2000). Saccadic inhibition and gaze contingent research paradigms. In A. Kennedy, R. Radach, D. Heller, \& J. Pynte (Eds.), Reading as a perceptual process. Amsterdam: North-Holland, pp. 119-145.

Tse, P.U., Sheinberg, D.L., \& Logothetis, N.K. (2002). Fixational eye movements are not affected by abrupt onsets that capture attention. Vision Research, 42, 1663-1669.

Tse, P.U., Sheinberg, D.L., \& Logothetis, N.K. (2003). Attentional enhancement opposite a peripheral flash revealed using change blindness. Psychological Science, 14, 91-99.

Winterson, B.J., \& Collewijn, H. (1976). Microsaccades during finely guided visuomotor tasks. Vision Research, 16, 1387-1390. 


\section{Acknowledgements}

We thank Jochen Laubrock for his contribution to this investigation, and Peter Tse and Bruce Bridgeman for their helpful comments on the manuscript. This research was supported by Deutsche Forschungsgemeinschaft (grants KL-955/3 and /6). 


\section{FIGURE CAPTIONS}

Figure 1. Microsaccade rate in a spatial cueing paradigm. (a) After 1 to 1.5 seconds a cue (white circle, $12.7^{\circ}$ eccentricity) was presented for $100 \mathrm{~ms}$ to the left or to the right indicating the most likely (validity $=80 \%$ ) position of a later target stimulus appearing after 1 to 1.5 seconds for $500 \mathrm{~ms}$ ( $12.7^{\circ}$ eccentricity). Participants were required to discriminate two possible target alternatives (a red vs. a green box) by pressing one of two buttons. Note, that this is an example of an invalid cue. (b) Temporal changes of microsaccade rate plotted separately for left and right cues. Areas of one between-subjects standard error are indicated by the shaded area around each curve. The white areas between rate curves in the post-cueintervals from $250 \mathrm{~ms}$ to $400 \mathrm{~ms}$ indicate significant differences. 
- Figure 1 -

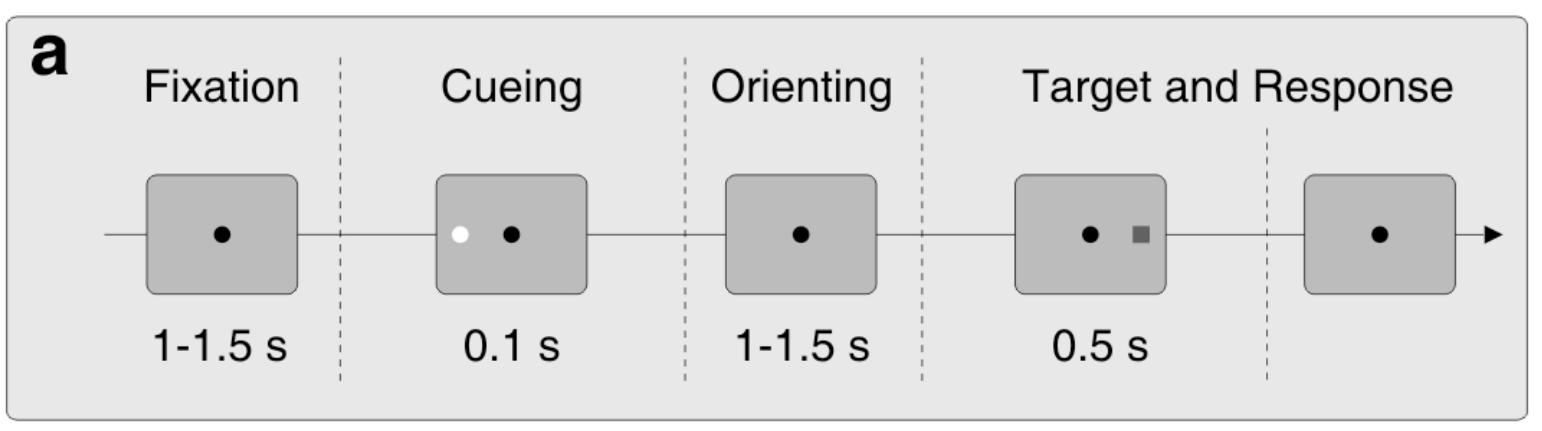

b

Cue right

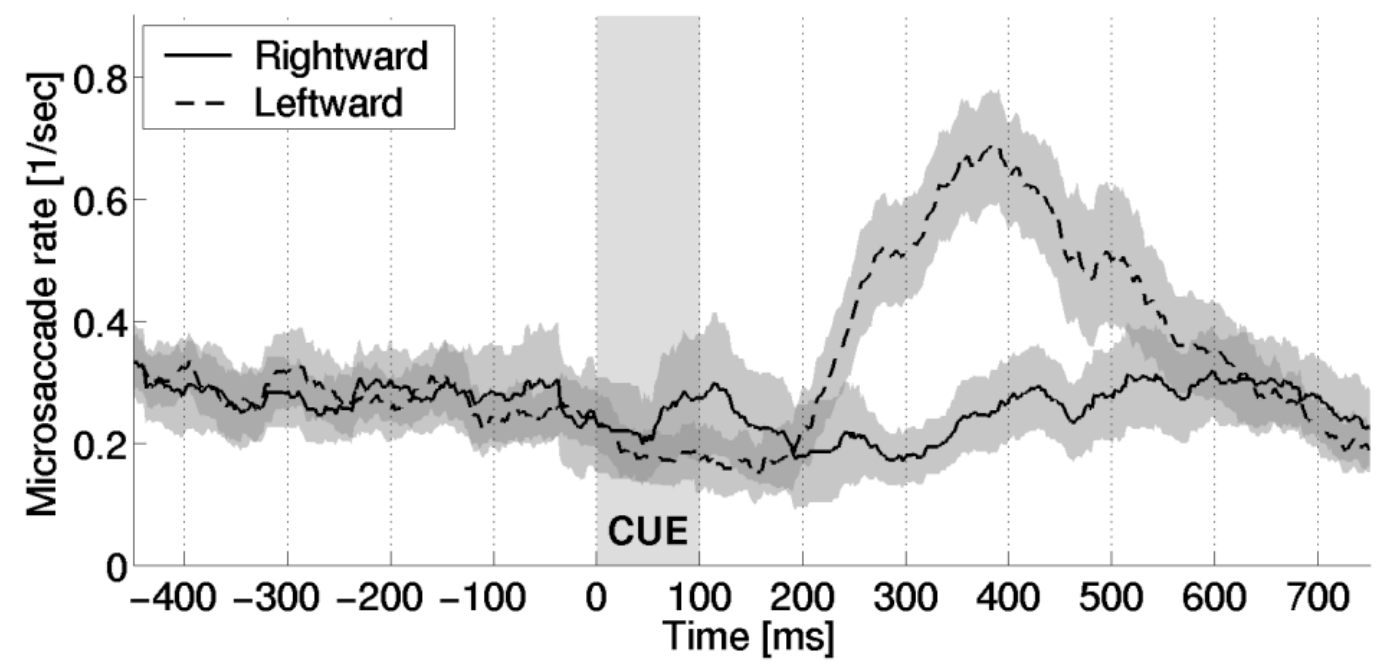

Cue left

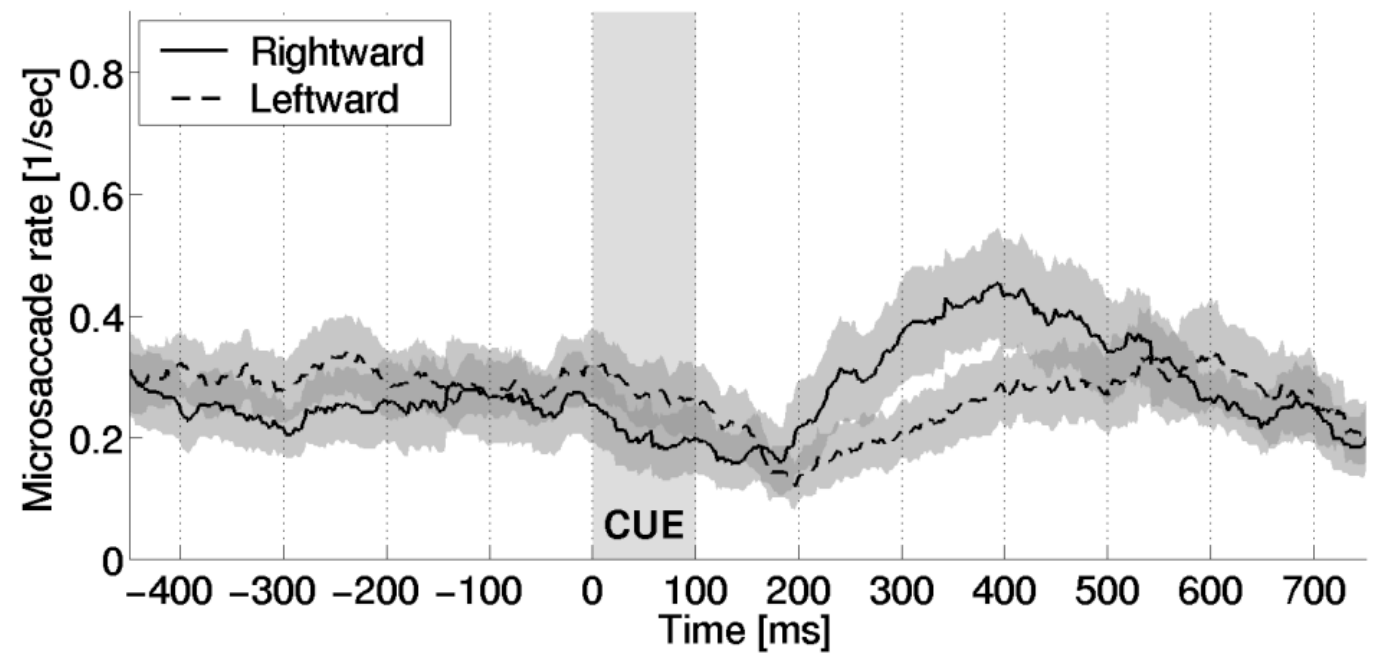

